\title{
Spin-isospin excitations as quantitative constraints for the tensor force
}

\author{
C. L. Bai, ${ }^{1,2}$ H. Q. Zhang, ${ }^{2}$ H. Sagawa, ${ }^{3}$ X. Z. Zhang, ${ }^{2}$ G. Colò, ${ }^{4}$ and F. R. Xu ${ }^{5}$ \\ ${ }^{1}$ School of Physics, Sichuan University, Chengdu 610065, China \\ ${ }^{2}$ China Institute of Atomic Energy, Beijing 102413, China \\ ${ }^{3}$ Center for Mathematics and Physics, University of Aizu, Aizu-Wakamatsu, Fukushima 965-8560, Japan \\ ${ }^{4}$ Dipartimento di Fisica, Università degli Studi di Milano and INFN, Sezione di Milano, IT-20133 Milano, Italy \\ ${ }^{5}$ School of Physics and State Key Laboratory of Nuclear Physics and Technology, Peking University, Beijing 100871, China
}

(Received 1 March 2011; published 19 May 2011)

\begin{abstract}
Gamow-Teller (GT) and charge-exchange spin-dipole (SD) excitation energies in ${ }^{90} \mathrm{Zr}$ and ${ }^{208} \mathrm{~Pb}$ are systematically studied to determine the appropriate magnitude of the tensor terms of the Skyrme interactions. We have found that the centroid energies of GT and SD excitations are sensitive to the adopted strengths of the triplet-even and triplet-odd tensor interactions. Especially, the $1^{-} \mathrm{SD}$ state plays a crucial role in constraining the triplet-even part while the triplet-odd part is related rather to the GT peaks. Among the $36 \mathrm{TIJ}$ parameter sets that include nonperturbatively the tensor terms, the four sets, T21, T32, T43, and T54, give reasonable results for the centroid energies in comparison with the experimental data. The sign and magnitude of the tensor terms are also discussed when these terms are added to the existing Skyrme interactions SGII and SLy5. The triplet-even strength can be constrained in a narrow range by using the available experimental data while further empirical data are needed to set a constraint on the triplet-odd term.
\end{abstract}

DOI: 10.1103/PhysRevC.83.054316

PACS number(s): 21.30.Fe, 21.10.Ky, 21.60.Jz, 23.20.-g

\section{INTRODUCTION}

Nuclear effective interactions such as the zero-range Skyrme forces [1] have been quite successful in describing many nuclear properties. These forces are fitted using empirical properties of uniform nuclear matter together with the masses and charge radii of selected reference nuclei. They describe in a reasonable way the global trends of the ground-state properties of nuclei in a wide range of the nuclear chart (e.g., binding energies, radii, and deformations). Yet, the tensor terms are not included in most of the Skyrme parameter sets that have been widely used. One of the current topics in mean-field theory is to fix the need and the properties of effective tensor terms by considering them within static mean field calculations [such as Hartree-Fock (HF) or HartreeFock-Bogoliubov (HFB) calculations], and also within the random-phase approximation (RPA) scheme devised for the excited states. Many groups have recently devoted attention to the role played by the zero-range tensor terms in nuclear structure problems (see Refs. [2-9]).

Different strategies are used to fix the tensor part of the interaction. One can be inspired by a bare or a G-matrix interaction $[2,10]$. Since the tensor force affects the spin-orbit splitting, another possibility is to add it to existing Skyrme sets and try to reproduce at best the evolution of single-particle states along isotopic or isotonic chains [4,5]. Another plausible way to obtain effective interactions including tensor terms, is a full variational procedure to fit the tensor and the central terms on equal footing [6]. All these attempts have produced results that are not very conclusive and are contradictory. It seems that the effects of the tensor force on the ground-state properties of nuclei, such as the total binding energy and radii, are not large enough to constrain well the tensor force. In addition, the mean-field models might not be enough to describe quantitatively the observed single-particle energies since other effects like the coupling with collective vibrations may be significant.

Recently, self-consistent HF + RPA schemes with tensor interactions have been developed [11-13]. The GT and chargeexchange $1^{+}$spin-quadrupole (SQ) transitions in ${ }^{90} \mathrm{Zr}$ and ${ }^{208} \mathrm{~Pb}$ have been studied in Refs. [11,12], whereas the noncharge-exchange multipole responses of several magic nuclei have been calculated in Ref. [13]. Very recently, the effects of the tensor force on the charge-exchange SD excitation in ${ }^{208} \mathrm{~Pb}$ have been studied and the results are compared to new experimental data [14]. In this latter study, it was shown that the tensor correlations have a clear multipole-dependent effect on the charge-exchange SD excitations [15]. This effect seems to have a very promising clear signature of the tensor force. In this paper we pursue our effort to constrain the effective Skyrmetype tensor interactions by using the available empirical information on the spin-dependent excitations such as GT and SD excitations in ${ }^{90} \mathrm{Zr}$ and ${ }^{208} \mathrm{~Pb}$.

With this aim, we examine all available Skyrme parameter sets with tensor terms that have been determined either with a variational procedure [6] or with a perturbative method. As criteria to test the tensor terms, we adopt the differences between the theoretical and experimental excitation energy of GT and SD states; in particular, we demand an accuracy of $2.5 \mathrm{MeV}$.

We sketch briefly our fully self-consistent HF + RPA model in Sec. II. In Sec. III, we report our GT- and SD-strength distributions in ${ }^{90} \mathrm{Zr}$ and ${ }^{208} \mathrm{~Pb}$ calculated by employing the TIJ parameter sets and compare the main peak energies with the experimental findings. In Sec. IV, we discuss the constraints on the strength of the triplet-even (TE) and tripletodd (TO) tensor terms added on top of the existing parameter sets SGII and SLy5 (using the GT and SD main peak energies in ${ }^{90} \mathrm{Zr}$ and ${ }^{208} \mathrm{~Pb}$ again). A summary is given in Sec. V. 


\section{THE HF + RPA CALCULATIONS WITH TENSOR FORCES}

The zero-range two-body tensor force we employ was originally proposed by Skyrme $[1,16]$ :

$$
\begin{aligned}
V^{T}= & \frac{T}{2}\left\{\left[\left(\sigma_{\mathbf{1}} \cdot \mathbf{k}^{\prime}\right)\left(\sigma_{\mathbf{2}} \cdot \mathbf{k}^{\prime}\right)-\frac{1}{3}\left(\sigma_{\mathbf{1}} \cdot \sigma_{2}\right) \mathbf{k}^{\prime 2}\right] \delta(r)\right. \\
& \left.+\delta(\mathbf{r})\left[\left(\sigma_{\mathbf{1}} \cdot \mathbf{k}\right)\left(\sigma_{\mathbf{2}} \cdot \mathbf{k}\right)-\frac{1}{3}\left(\sigma_{\mathbf{1}} \cdot \sigma_{2}\right) \mathbf{k}^{2}\right]\right\} \\
& +\frac{U}{2}\left\{\left(\sigma_{1} \cdot \mathbf{k}^{\prime}\right) \delta(\mathbf{r})\left(\sigma_{2} \cdot \mathbf{k}\right)+\left(\sigma_{2} \cdot \mathbf{k}^{\prime}\right) \delta(\mathbf{r})\left(\sigma_{1} \cdot k\right)\right. \\
& \left.-\frac{2}{3}\left[\left(\sigma_{1} \cdot \sigma_{2}\right) \mathbf{k}^{\prime} \cdot \delta(\mathbf{r}) \mathbf{k}\right]\right\} .
\end{aligned}
$$

In the above expression, the operator $\mathbf{k}=\left(\nabla_{\mathbf{1}}-\nabla_{\mathbf{2}}\right) / 2 i$ acts on the right and $\mathbf{k}^{\prime}=-\left(\nabla_{1}^{\prime}-\nabla_{2}^{\prime}\right) / 2 i$ acts on the left. The parameters $T$ and $U$ denote the strengths of TE and TO tensor terms, respectively.

In spherical symmetry, the tensor contribution to the Skyrme energy functional is expressed by means of the spin-orbit density $J_{q}$ as

$$
J_{q}=\frac{1}{4 \pi r^{3}} \sum_{i}\left(2 j_{i}+1\right)\left[j_{i}\left(j_{i}+1\right)-l_{i}\left(l_{i}+1\right)-3 / 4\right] R_{i}^{2}(r) \text {, }
$$

where $q=n(p)$ labels neutron (or proton). The associated part of energy-density functional is given by

$$
H^{t}=\sum_{t=0,1} \frac{1}{2} C_{t}^{J} \mathbf{J}_{\mathbf{t}}^{\mathbf{2}}
$$

where $\mathbf{J}_{\mathbf{t}=\mathbf{0}}=\mathbf{J}_{\mathbf{n}}+\mathbf{J}_{\mathbf{p}}$ and $\mathbf{J}_{\mathbf{t}=\mathbf{1}}=\mathbf{J}_{\mathbf{n}}-\mathbf{J}_{\mathbf{p}}$. The effective coupling constants receive contribution from both the nonlocal central terms and the tensor terms, namely

$$
C_{t}^{J}=A_{t}^{J}+B_{t}^{J}
$$

with

$$
\begin{aligned}
& A_{0}^{J}=\frac{1}{8} t_{1}\left(\frac{1}{2}-x_{1}\right)-\frac{1}{8} t_{2}\left(\frac{1}{2}+x_{2}\right), \\
& A_{1}^{J}=\frac{1}{16}\left(t_{1}-t_{2}\right), \\
& B_{0}^{J}=\frac{5}{48}(T+3 U), \\
& B_{1}^{J}=\frac{5}{48}(U-T) .
\end{aligned}
$$

Then Eq. (3) can be written in the notation used in Ref. [10]:

$$
\Delta H=\frac{1}{2} \alpha\left(J_{n}^{2}+J_{p}^{2}\right)+\beta J_{n} J_{p}
$$

with

$$
\begin{aligned}
& \alpha=C_{0}^{J}+C_{1}^{J}, \\
& \beta=C_{0}^{J}-C_{1}^{J} .
\end{aligned}
$$

It should be noted that $J_{q}$ is almost negligible in a spin-orbit saturated nuclei in which both spin-orbit partners are filled.

The operator for GT transitions is defined as

$$
\hat{O}_{\mathrm{GT} \pm}=\sum_{i m} t_{ \pm}^{i} \sigma_{m}^{i}
$$

and the spin-dependent charge-exchange SD operator is given as

$$
\hat{O}_{\mathrm{SD} \pm}^{J}=\sum_{i M} t_{ \pm}^{i} r_{i}^{l}\left[Y_{l}^{i}(\hat{r}) \sigma^{i}\right]^{\mathrm{JM}}
$$

in terms of the standard isospin operators, $t_{ \pm}=\frac{1}{2}\left(t_{x} \pm i t_{y}\right)$. For these excitations we have developed a self-consistent chargeexchange HF + RPA method based on the Skyrme force. In this model, both the two-body tensor terms and the two-body spinorbit interaction are included to make the calculation fully selfconsistent. We start by solving the HF equations in coordinate space with a large radial mesh extending up to $20 \mathrm{fm}$ (with a step of $0.1 \mathrm{fm}$ ). After the Skyrme HF potential is calculated, the single-particle wave functions of the occupied and unoccupied levels are obtained by using a harmonic-oscillator basis that extends up to the maximum major quantum number $N_{\max }=10$ and 12 for ${ }^{90} \mathrm{Zr}$ and ${ }^{208} \mathrm{~Pb}$, respectively. In the charge-exchange RPA, the $t_{-}$and $t_{+}$channels are coupled and the corresponding eigenstates emerge from a single diagonalization of the RPA matrix.

\section{RESULTS OF TI $J$ FORCES ON THE GT AND CHARGE-EXCHANGE SD TRANSITIONS $\mathrm{OF}^{90} \mathrm{Zr}$ AND ${ }^{208} \mathrm{~Pb}$}

In Ref. [6], 36 different Skyrme sets, the so-called TI J family, were proposed; they include the central and tensor terms on equal footing and all parameters were determined by a variational procedure analogous to the one employed for the SLy interactions [17]. The strengths of the TE and TO tensor forces are varied in a wide range by means of the conditions:

$$
\alpha=(J-2) 60 \mathrm{MeV} \mathrm{fm}^{5}, \quad \beta=(I-2) 60 \mathrm{MeV} \mathrm{fm}^{5},
$$

with $I, J=(1, \ldots, 6)$. Then the ground-state properties of the selected nuclei were fitted by changing the central part of the Skyrme interactions.

We have studied the GT and charge-exchange SD transitions in ${ }^{90} \mathrm{Zr}$ and ${ }^{208} \mathrm{~Pb}$ by employing the 36 parameter sets of the TI $J$ family. The strengths are broadly distributed and finding a criterion to compare theory and experiment is not straightforward. We have decided to consider for each force and each strength function the energy region in which the strength is larger than one-half of its value in the main peak. In this energy region, we have calculated the centroid energy defined as the ratio between energy-weighted strength and nonenergy-weighted strength [i.e., $m(1) / m(0)]$. These centroid energies labeled as $\mathrm{E}_{\text {th }}$ are compared with the experimental result $\mathrm{E}_{\exp }$ (in this paper, the excitation energies are calculated with respect to the ground state of the mother nuclei). The difference $\delta E=\left|E_{\mathrm{th}}-E_{\text {exp }}\right|$ is treated as a benchmark in order to choose the appropriate parameter sets. We consider the total SD- and the GT-strength functions in ${ }^{90} \mathrm{Zr}$, whereas in ${ }^{208} \mathrm{~Pb}$, besides the GT- and the total SD-strength functions, the SD $1^{-}$strength is also considered. We find that the four parameter sets, T21, T32, T43, and T54, meet the criteria of having $\delta E \leqslant 2.5 \mathrm{MeV}$ for all modes, as shown in Table I.

It is convenient that the results change only slightly (and the selected sets remain the same) because then the main-peak 
TABLE I. The calculated peak energies of the SD and GT strengths in ${ }^{90} \mathrm{Zr}$ and ${ }^{208} \mathrm{~Pb}$ obtained by using the four interactions that reproduce the experimental data $[14,18,19]$ within an accuracy of $2.5 \mathrm{MeV}$. See the text for a discussion.

\begin{tabular}{|c|c|c|c|c|c|c|c|c|c|c|}
\hline & \multicolumn{5}{|c|}{${ }^{90} \mathrm{Zr}$} & \multicolumn{5}{|c|}{${ }^{208} \mathrm{~Pb}$} \\
\hline & $0^{-}$ & $1^{-}$ & $2^{-}$ & total SD & GT & $0^{-}$ & $1^{-}$ & $2^{-}$ & total SD & GT \\
\hline T32 & 39.0 & 23.8 & 25.4 & 24.3 & 15.9 & 39.4 & 23.4 & 25.3 & 23.3 & 17.4 \\
\hline $\mathrm{T} 43$ & 38.6 & 24.3 & 25.3 & 24.9 & 16.2 & 37.7 & 24.0 & 25.4 & 23.6 & 17.2 \\
\hline T54 & 38.3 & 24.5 & 25.4 & 25.2 & 16.2 & 37.1 & 23.8 & 25.4 & 23.5 & 16.7 \\
\hline
\end{tabular}

energies are used as a benchmark instead of the centroid energies. Moreover, the set T43 that was used satisfactorily in Ref. [15] respects all the criteria.

From the results listed in Table I, one can see that in the experimental results for ${ }^{208} \mathrm{~Pb}$, the peak energy of $0^{-}$is the highest. Then, the $2^{-}$peak lies in the middle and the lowest peak is associated with the $1^{-}$strength. The SD $1^{-}$mode in ${ }^{208} \mathrm{~Pb}$ plays an important role to exclude many parameter sets. The calculated energy of the $0^{-}$strength is somewhat higher than the experimental result. This might be due to the fact that the values of $U$ in the four parameter sets are always negative (see Eqs. (6) and (8) of Ref. [15]). Furthermore, the GT and SD excitation energies can give a strong constraint on the Skyrme parameters. In fact, one can find in Fig. 1 of Ref. [6] that the values of $C_{1}^{J}$ for all four parameter sets are $-30 \mathrm{MeV} \mathrm{fm}^{5}$.

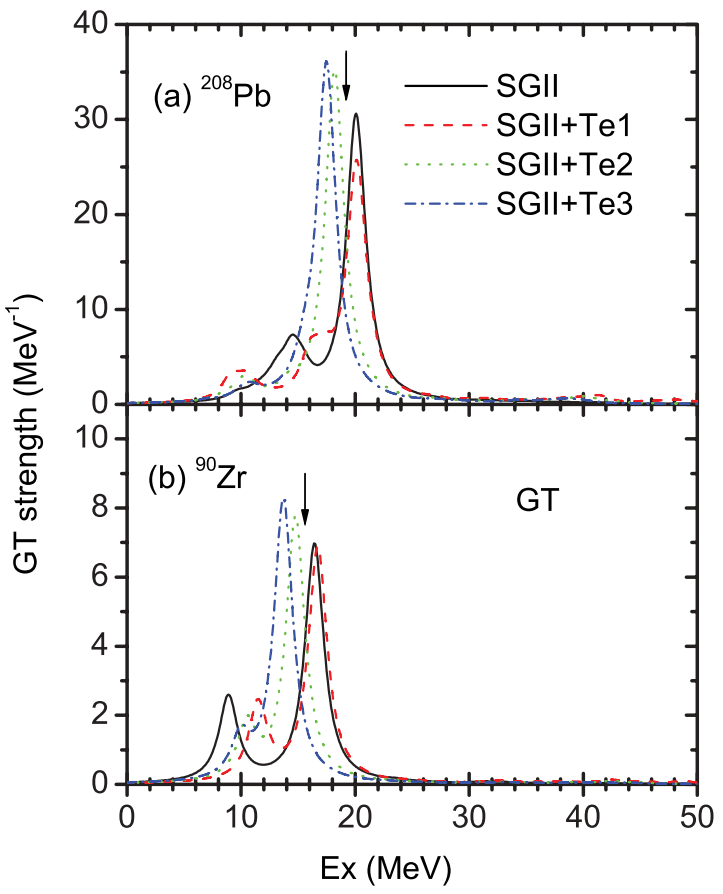

FIG. 1. (Color online) The charge-exchange GT-strength distributions of ${ }^{90} \mathrm{Zr}$ and ${ }^{208} \mathrm{~Pb}$ calculated with SGII, SGII + Te1, SGII + $\mathrm{Te} 2$, and SGII $+\mathrm{Te} 3$. The experimental peak energies of the GT resonances are shown by the arrows. The discrete RPA results have been smoothed by using a Lorentzian averaging with a width of $1 \mathrm{MeV}$. See the text for more details.

\section{CONSTRAINTS FOR TENSOR INTERACTIONS WITH SGII AND SLY5}

In this Section, we study the effect on GT and SD states produced by the tensor interactions that are obtained by adding tensor terms on top of the existing parameter sets, SGII and SLy5. In particular, we calculate the GT and SD states by adopting tensor terms characterized by a sufficiently wide range for $T$ and $U$. We then constrain the tensor parameters $T$ and $U$ by requiring $\delta E \leqslant 2.5 \mathrm{MeV}$ (see the previous Section for the definition of $\delta E$ ).

We have tried to add tensor terms on top of the force SGII, whose central part is fitted by taking care of the correct sign of the spin-isospin Landau parameter, $G_{0}^{\prime}$ (the force must be strongly repulsive in this channel). Since there is some ambiguity in the sign of $U$ (see below), we choose three points in the $(T, U)$ plane characterized by the values $(T, U)=(500$, $-350),(600,0)$, and $(650,200)$ in order to study in detail the GT and SD transitions in ${ }^{208} \mathrm{~Pb}$. The parameters are labeled as SGII + Te1, SGII + Te2, and SGII + Te3, respectively. In Fig. 1, the results of the GT-strength distribution are shown in the cases of ${ }^{90} \mathrm{Zr}$ and ${ }^{208} \mathrm{~Pb}$. The solid lines labeled by "SGII" mean that the tensor force is not included (neither in HF nor in RPA). The dashed, dotted, and dashed-dotted lines show the results with the included tensor interactions SGII + $\mathrm{Te} 1$, SGII $+\mathrm{Te} 2$, and SGII $+\mathrm{Te} 3$, respectively. As far as the GT peak energies are concerned, the three tensor interactions provide results that are within the $2.5 \mathrm{MeV}$ interval for both ${ }^{90} \mathrm{Zr}$ and ${ }^{208} \mathrm{~Pb}$. Among the three interactions, $\mathrm{SGII}+\mathrm{Te} 2$ gives the best results.

The strength distributions for the case of charge-exchange SD transitions in ${ }^{90} \mathrm{Zr}$ and ${ }^{208} \mathrm{~Pb}$ calculated by using the three parameter sets are shown in Fig. 2. The strengths of SD $0^{-}$, $1^{-}$, and $2^{-}$modes, as well as the total strength, are shown in panels (a), (b), (c), and (d), respectively. The experimental SD data are only available for the total strength in ${ }^{90} \mathrm{Zr}$ while each multipole was separated by the multipole-decomposition analysis in the case of ${ }^{208} \mathrm{~Pb}$ [14]. For ${ }^{208} \mathrm{~Pb}$, the $1^{-}$and the total strength distributions are shown in panels (b) and (d), respectively. The tensor force obviously improves the agreement of the theoretical results with the experimental. In panel (c), the calculated result is slightly shifted upward by the tensor interaction closer to the observed $2^{-}$peak at the excitation energy around $25 \mathrm{MeV}$. It is seen that for the $0^{-}$ state [panel (a)] the SGII + Te3 set with a positive value of $U$ is preferable among the three tensor interactions. 

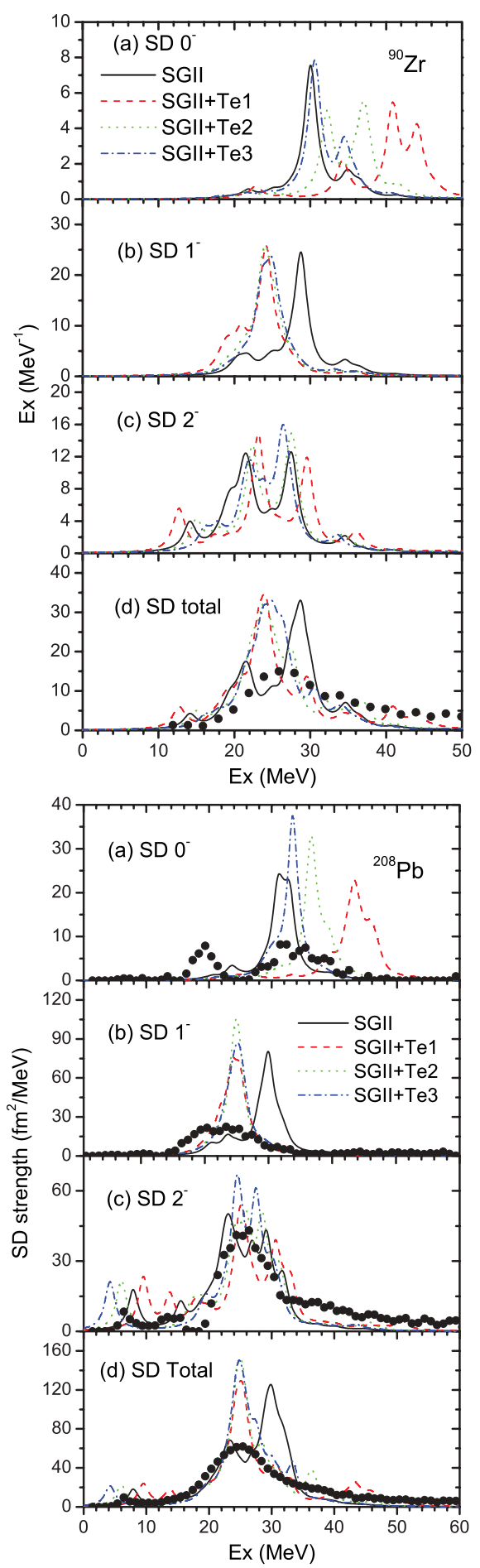

FIG. 2. (Color online) The charge-exchange SD-strength distribution of ${ }^{90} \mathrm{Zr}$ and ${ }^{208} \mathrm{~Pb}$ calculated with SGII, SGII + Te1, SGII + $\mathrm{Te} 2$, and SGII + Te3 interactions. The discrete RPA results have been smoothed by using a Lorentzian averaging with a width of $1 \mathrm{MeV}$ and compared with experimental data [14]. See the text for more details.

In Fig. 3, we display the results of a more systematic study of possible values for the $T$ and $U$ parameters that can respect the constraint $\delta E \leqslant 2.5 \mathrm{MeV}$ for the different modes (with the same definition as in the previous section). The lines with filled circles are the lower and upper limits for GT centroid

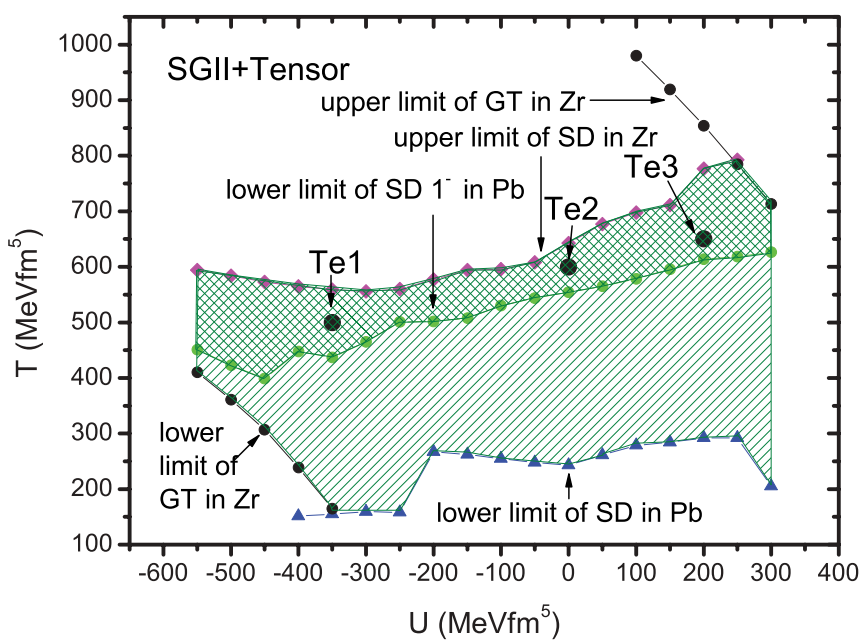

FIG. 3. (Color online) The region of $T$ and $U$ values constrained by the criterion $\delta E \leqslant 2.5 \mathrm{MeV}$ for the GT and total SD centroid energies in ${ }^{90} \mathrm{Zr}$ and ${ }^{208} \mathrm{~Pb}$ and for the SD $1^{-}$centroid energy in ${ }^{208} \mathrm{~Pb}$. The points $\mathrm{Te} 1, \mathrm{Te} 2, \mathrm{Te} 3$, and $\mathrm{Te} 4$ correspond to specific tensor forces added on top of SGII and used to calculate the strength distributions in the previous figures. See the text for more details.

energies in ${ }^{90} \mathrm{Zr}$. The line with triangles is the lower limit for the centroid of the total SD strength in ${ }^{208} \mathrm{~Pb}$ while the line with diamonds shows the upper limit obtained from the centroid of the total SD strength in ${ }^{90} \mathrm{Zr}$. As it can be seen, the value of $T$ is constrained to be between 150 and $750 \mathrm{MeV} \mathrm{fm}^{5}$ by the GT and SD centroids while they give essentially no constraint for the value of $U$. It has been noticed that if the tensor force is not included in the calculation, the calculated $1^{-}$peak lies at a much higher energy than the experimental finding in ${ }^{208} \mathrm{~Pb}$, as seen in Fig. 2(b). Thus, the $1^{-}$state provides a strong constraint for the tensor interactions, namely, a large and positive value for $T$ is required to match the experiment. The line with circles in the shaded area corresponds to the lower limit for the $1^{-}$ centroid energy in ${ }^{208} \mathrm{~Pb}$. Adding the constraint from the $1^{-}$ mode, the value of $T$ is limited within an area specified by $-50+T_{0}<T<50+T_{0}$, where $T_{0}$ is increasing from 500 to $700 \mathrm{MeV} \mathrm{fm}{ }^{5}$ depending on the value of $U$, as shown in the double-shaded area.

A difference of the Skyrme force in the central part may produce different constraints on the strength of TE and TO tensor interactions. To illustrate this point, we now add tensor terms on top of SLy5 (which is fitted with a similar protocol as the TI $J$ family).

In Fig. 4, in a similar style as in the previous figure, we show the lines, which are obtained with the same constraint, $\delta E \leqslant 2.5 \mathrm{MeV}$, for the GT and SD centroid energies. As it was pointed out in the case of SGII, the $1^{-}$peak in ${ }^{208} \mathrm{~Pb}$ calculated with SLy5 (no tensor) is much higher than the experimental finding and it gives a strong constraint especially for the $T$ value of the tensor force when this is added. When the SD $1^{-}$centroid energy in ${ }^{208} \mathrm{~Pb}$ is included in the constraints, the acceptable area for the parameters is roughly triangle-shaped. $U$ can range from -300 to $-50 \mathrm{MeV} \mathrm{fm}^{5}$ and the minimum value for $T$ is $450 \mathrm{MeV} \mathrm{fm}^{5}$. The maximum value of $T$ depends on $U$ (the absolute maximum being close to $650 \mathrm{MeV} \mathrm{fm}^{5}$ ). In 


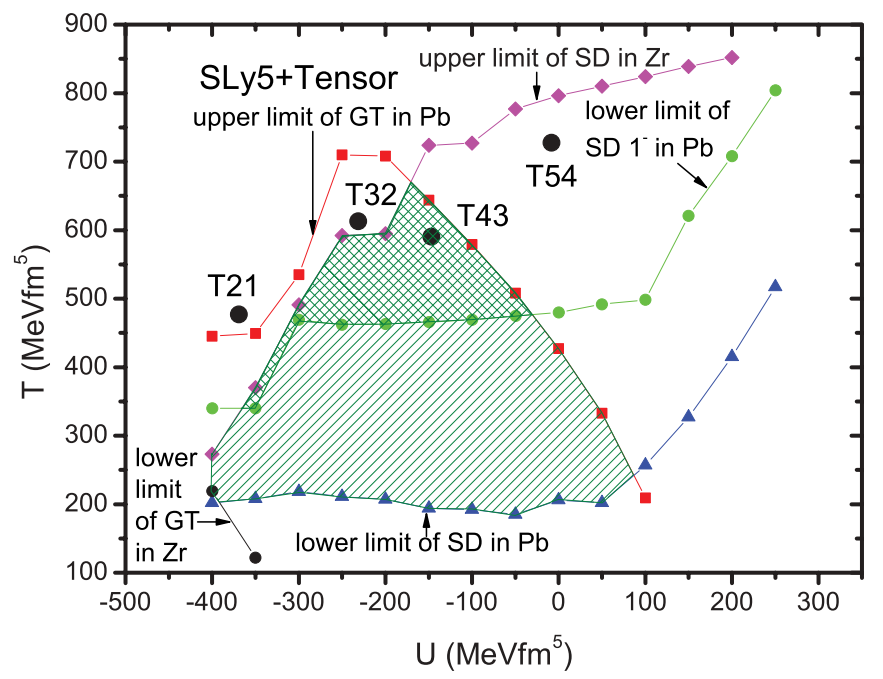

FIG. 4. (Color online) The same as Fig. 3 but with the tensor force added on top of SLy5. The points correspond to the forces TI J selected in Sec. III (however, the central part is different from that of SLy5, so their position in this plot is only indicative). See the text for more details.

the same figure, we also show the position of the forces TIJ selected in Sec. III. However, the central part is different from that of SLy5, so their position in this plot is only indicative.

\section{SUMMARY}

In summary, we have studied the GT and SD transitions in ${ }^{90} \mathrm{Zr}$ and ${ }^{208} \mathrm{~Pb}$ in order to constrain effectively the tensor terms of the Skyrme force. We have chosen the criterion that the centroid energy differences, $\delta E$ (between the theoretical and experimental values), should be smaller than the value $\delta E=2.5 \mathrm{MeV}$ for the case of the GT and total SD strengths in ${ }^{90} \mathrm{Zr}$ and for the $1^{-}$SD strength in ${ }^{208} \mathrm{~Pb}$.
We have systematically studied the 36 parameter sets in the TI $J$ family. We have found that the GT strength distribution, the total SD, and the SD $1^{-}$and $2^{-}$are much affected by the tensor interaction. Especially, the SD $1^{-}$state in ${ }^{208} \mathrm{~Pb}$ plays a crucial role to constrain the tensor parameters. It is found that the four interactions, $\mathrm{T} 21, \mathrm{~T} 32$, T43, and T54, meet the requested criteria.

We have also built tensor forces adding them on the top of the existing parameter sets SGII and SLy5. It is shown that the above-defined constraints restrict the acceptable values for the parameters $T$ and $U$ within a rather narrow region. Firstly, the value of $T$ should be positive and have a value around $550 \mathrm{MeV} \mathrm{fm}{ }^{5}$ (depending somehow mildly on the central part of the Skyrme force). This is mainly required by the matching with the SD $1^{-}$mode. Secondly, the accepted value of $U$ can be constrained by the GT peak energy, although it somewhat depends more on the choice of the central part of the Skyrme interaction. With the parameter set SGII, a wide range of $U$ values is acceptable satisfying the criterion $\delta E<2.5 \mathrm{MeV}$. With SLy5, the value of $U$ should be negative as $U \sim-(180 \pm 100) \mathrm{MeV} \mathrm{fm}^{5}$ due to the requirement of reproducing the GT centroid energy of ${ }^{208} \mathrm{~Pb}$. Thus, the triplet-odd tensor strength, $U$, is still not well constrained. Since the SD $0^{-}$excitation energy is very sensitive to the value of $U$, further experimental investigation will give a more strict constraint for the realistic value of $U$.

\section{ACKNOWLEDGMENTS}

This work is supported by the National Natural Science Foundation of China under Grant Nos. 10875172, 10275092, and 10675169, the National Key Basic Research Program of China under Grant No. 2007CB815000, and Financial Supports from Sichuan University (Project No. 2010SCU11086). Partial support from the Italian research project (PRIN) named "Many-body theory of nuclear systems and implications on the physics of neutron stars" is also acknowledged.
[1] T. H. R. Skyrme, Nucl. Phys. 9, 615 (1959).

[2] B. A. Brown, T. Duguet, T. Otsuka, D. Abe, and T. Suzuki, Phys. Rev. C 74, 061303(R) (2006).

[3] J. Dobaczewski, in Proceedings of the Third ANL/MSU/JINA/INT RIA Workshop, edited by T. Duguet, H. Esbensen, K. M. Nollett, and C. D. Roberts (World Scientific, Singapore, 2006).

[4] G. Colò, H. Sagawa, S. Fracasso, and P. F. Bortignon, Phys. Lett. B 646, 227 (2007).

[5] D. M. Brink and Fl. Stancu, Phys. Rev. C 75, 064311 (2007).

[6] T. Lesinski, M. Bender, K. Bennaceur, T. Duguet, and J. Meyer, Phys. Rev. C 76, 014312 (2007).

[7] M. Grasso, Z. Y. Ma, E. Khan, J. Margueron, and N. Van Giai, Phys. Rev. C 76, 044319 (2007).

[8] W. Zou, G. Coló, Z. Y. Ma, H. Sagawa, and P. F. Bortignon, Phys. Rev. C 77, 014314 (2008).

[9] M. Zalewski, J. Dobaczewski, W. Satula, and T. R. Werner, Phys. Rev. C 77, 024316 (2008).
[10] Fl. Stancu, D. M. Brink, H. Flocard, Phys. Lett. B 68, 108 (1977).

[11] C. L. Bai, H. Sagawa, H. Q. Zhang, X. Z. Zhang, G. Colò, and F. R. Xu, Phys. Lett. B 675, 28 (2009).

[12] C. L. Bai, H. Q. Zhang, X. Z. Zhang, F. R. Xu, H. Sagawa, and G. Colò, Phys. Rev. C 79, 041301(R) (2009).

[13] L. Cao, G. Coló, H. Sagawa, P. F. Bortignon, and L. Sciacchitano, Phys. Rev. C 80, 064304 (2009).

[14] T. Wakasa, arXiv:1004.5220 [nucl-ex].

[15] C. L. Bai, H. Q. Zhang, H. Sagawa, X. Z. Zhang, G. Colò, and F. R. Xu, Phys. Rev. Lett. 105, 072501 (2010).

[16] T. H. R. Skyrme, Philos. Mag. 1, 1043 (1956).

[17] E. Chabanat, P. Bonche, P. Haensel, J. Meyer, and R. Schaeffer, Nucl. Phys. A 627, 710 (1997).

[18] T. Wakasa et al., Phys. Rev. C 55, 2909 (1997); H. Akimune et al., ibid. 52, 604 (1995).

[19] H. Sagawa, S. Yoshida, X. R. Zhou, K. Yako, and H. Sakai, Phys. Rev. C 76, 024301 (2007). 